\title{
Novel Gold(I) and Silver(I) Complexes of Phosphorus-1,1,-dithiolates and Molecular Structure of $\left[\mathrm{O}, \mathrm{O}^{\prime}-(\text { Bornyl) })_{2} \mathrm{PS}_{2}\right] \mathrm{H}_{3} \mathrm{NC}\left(\mathrm{CH}_{3}\right)_{3}$
}

\author{
Samet Solak', Cemal Aydemir ${ }^{1}$, Mehmet Karakus ${ }^{*}$ and Peter Lönnecke ${ }^{2}$
}

\begin{abstract}
Background: The novel chiral phosphorus-1,1-dithiolates [4- $\left.\mathrm{CH}_{3} \mathrm{OC}_{6} \mathrm{H}_{4} \mathrm{P}(\mathrm{S})(\mathrm{OR}) \mathrm{S}\right]-\left[\mathrm{H}_{3} \mathrm{NC}\left(\mathrm{CH}_{3}\right)_{3}\right]^{+}$were synthesized by the reaction of $\left.\left[\mathrm{RPS}_{2}\right)\right]_{2}\left(\mathrm{R}=4-\mathrm{MeOC}_{6} \mathrm{H}_{4}\right)$ or $\mathrm{P}_{2} \mathrm{~S}_{5}$ and the respective alcohol $\mathrm{ROH}(\mathrm{R}=$ myrtanyl, 2-naphthylethyl, myrtenyl, borneol) in toluene. The reaction of phosphorus-1,1-dithiolates 1-4 and $\mathrm{Au}($ tht $) \mathrm{Cl}, \mathrm{AuClPPh}_{3} \mathrm{Or} \mathrm{AgNO}_{3}$ and $\mathrm{PPh}_{3}$ gave rise to gold(I)- and silver(I)-complexes in THF. All compounds have been characterised by elemental analyses, IR, NMR $\left({ }^{1} \mathrm{H}-,{ }^{13} \mathrm{C}\right.$ - and $\left.{ }^{31} \mathrm{P}-\right)$ spectroscopy as well as MS measurements. Optical rotation values confirmed the chirality of the compounds. The Compound 4 has been characterized structurally by $\mathrm{X}$-ray crystallography.

Results: Phosphorus-1,1,-dithiolate compounds were formed as liquids and were treated with suitable amine in order to convert them to their salts $1-4$. They have been successfully characterized spectroscopically $\left(\mathbb{R},{ }^{1} \mathrm{H},{ }^{13} \mathrm{C},{ }^{31} \mathrm{P}\right.$ NMR) as well as mass spectra. The compound 4 has been also structurally by X-ray crystallography. The compound 4 crystallizes in the orthorhombic space group P2(1)2(1)2(1) with Z =4. Compounds containing phosphorus and sulfur donor atoms are excellent ligands due to offering many metal complexes especially group 11-12 metals. The synthesis of gold(I) and silver(I) complexes with chiral phosphorus-1,1,-dithiolate and triphenylphosphine have been described and investigated.

Conclusions: In the present work, we report the synthesis, charactreization of the chiral phosphorus-1,1-dithiolate ligands and preparing the gold(I) and silver(I) phosphorus-1,1-dithiolate or S-donor with phosphine complexes. The molecular structure of the Compound 4 was determined by $X$-ray diffraction. Due to an easy synthesis method of phosphorus-1,1-dithiolate compounds and a good complexion reagent, it is possible the improvement of the collecting metallic gold or silver from the minerals. When the more ionic salt of phosphorus-1,1-dithiolate compounds were prepared in this way, the water can be used as a cheap solvent. As a result, it can be an alternatively method for the collecting metallic gold or silver from the minerals in future.
\end{abstract}

Keywords: Phosphorus-1,1-dithiolate, Chiral, Silver(I) and gold(I) complexes

\section{Background}

Phosphorus-1,1'-dithiolate ligands are an important class in organophophorus chemistry [1,2]. To date, many phosphorus-1,1'-dithiolate have been synthesized and widely utilised in agricultural, medicinal and technological fields[1-4]. Since the discovery of Lawesson reagent's and its anologue, they have been used a thionation reagent in organic chemistry and also considerable number of dithiophosphonates and their metal complexes have been

\footnotetext{
* Correspondence: mkarakus@pau.edu.tr

${ }^{1}$ Department of Chemistry, Faculty of Arts \& Sciences, Pamukkale University, Kinikli 20075, Denizli, Turkey

Full list of author information is available at the end of the article
}

synthesized [5-29]. For example, dithiophosphonates which are derivatives of phosphorus-1,1'-dithiolate ligands are not commercially available but they can be easily synthesized by the reaction of Lawesson's reagent or Ferrocenyl Lawesson's reagent and the respective alcohols or amines due to a ring opening reaction by nucleophilic attack. The application of metal complexes in the field of medicinal, bioinorganic and bioorganic chemistry has become important in recent decades. For example, some complexes of gold(I), such as Auranofin and some related complexes have been used in the treatment of severe rheumathoidal arthritis [30]. Although there are many gold(I), silver(I) and copper(I) complexes of dithiophosphonates 
[8-12,22,24], chiral dithiophosphonates and their metal complexes are rare. Recently, a few gold(I) complexes with chiral dithiophosphonates have been reported in our laboratory [5,6].

In the present work, we report mono and dinuclear metal complexes with chiral dithiophosphonates and also triphenylphosphine complexes. All compounds were characterized by elemental analyses, IR, NMR $\left({ }^{1} \mathrm{H}-,{ }^{13} \mathrm{C}-,{ }^{31} \mathrm{P}-\right)$ spectroscopy as well as MS measurements.

\section{Results and discussion}

Chiral phosphorus-1,1-dithiolate ligands have been synthesized from Lawesson's reagent and chiral hydroxyl compounds (Scheme 1).

O-dithiophosphonic acid derivatives of phosphorus-1,1,dithiolate were formed as liquids and were treated with suitable amine in order to convert them to their salts 1-4. The ${ }^{31} \mathrm{P}$ NMR spectra of the ligands $\mathbf{1 - 4}$ were measured in $\left[D_{6}\right]$ DMSO and showed one signal as expected. The synthesis and X-ray determination of the compound $\mathbf{2}$ was reported by Solak et. al. [7] but the spectral studies of the compound $\mathbf{2}$ were not. All ligands 1-4 and gold(I) complexes reported here have been characterised by elemental analysis, IR, NMR and mass spectrocopies. An analogue (triethyl ammonium -O,O'-diborneildithiophosphate) of the compound $\mathbf{4}$ was sythesized by Ohta et. al. [31]. In this study, a single crytal structural and full spectroscopically studies of the compound 4, tert-butylammonium -O,O'diborneildithiophosphate, also performed here.

The synthesis of gold(I) and silver(I) complexes with chiral phosphorus-1,1,-dithiolate and triphenylphosphine have been described and also characterized by elemental analyses, IR, NMR and MS spectroscopies (Additional file 1). The synthesis mononuclear and dinuclear gold(I) complexes were summarized in the Scheme 2. The ligands 1, 2 and 3 were reacted with $\mathrm{AuCl}\left(\mathrm{PPh}_{3}\right)$ in order
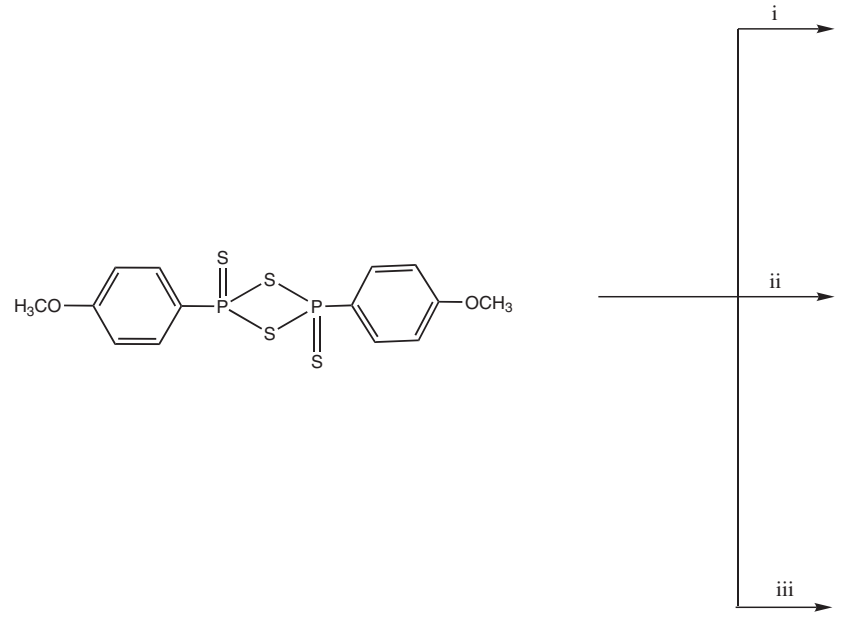

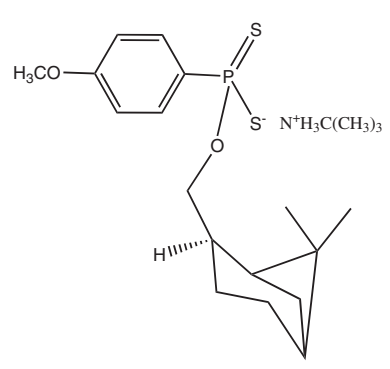

(1)

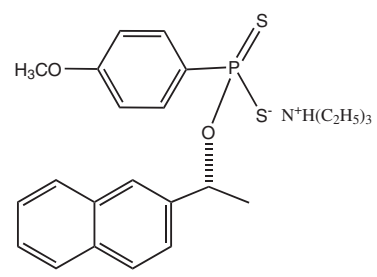

(2)

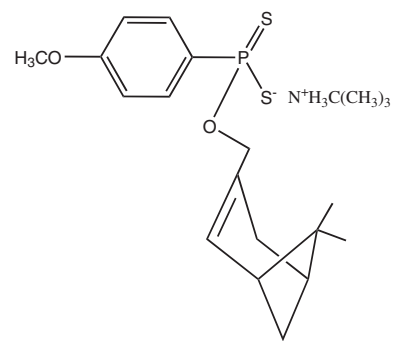

(3)

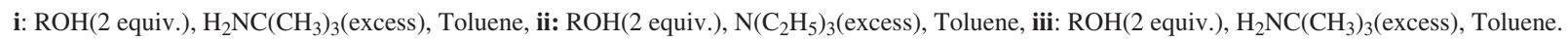

$\mathrm{ROH}$ :<smiles>C[C@@H](O)c1ccc2ccccc2c1</smiles>

(S)-(-)-1-(2-Naphthyl)ethanol

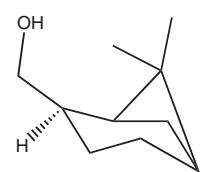

(1S,2S,5S)- (-)-myrtanol

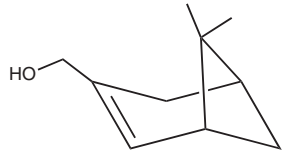

(1R)-(-)-myrtenol

Scheme 1 Synthesis of 1-3. 


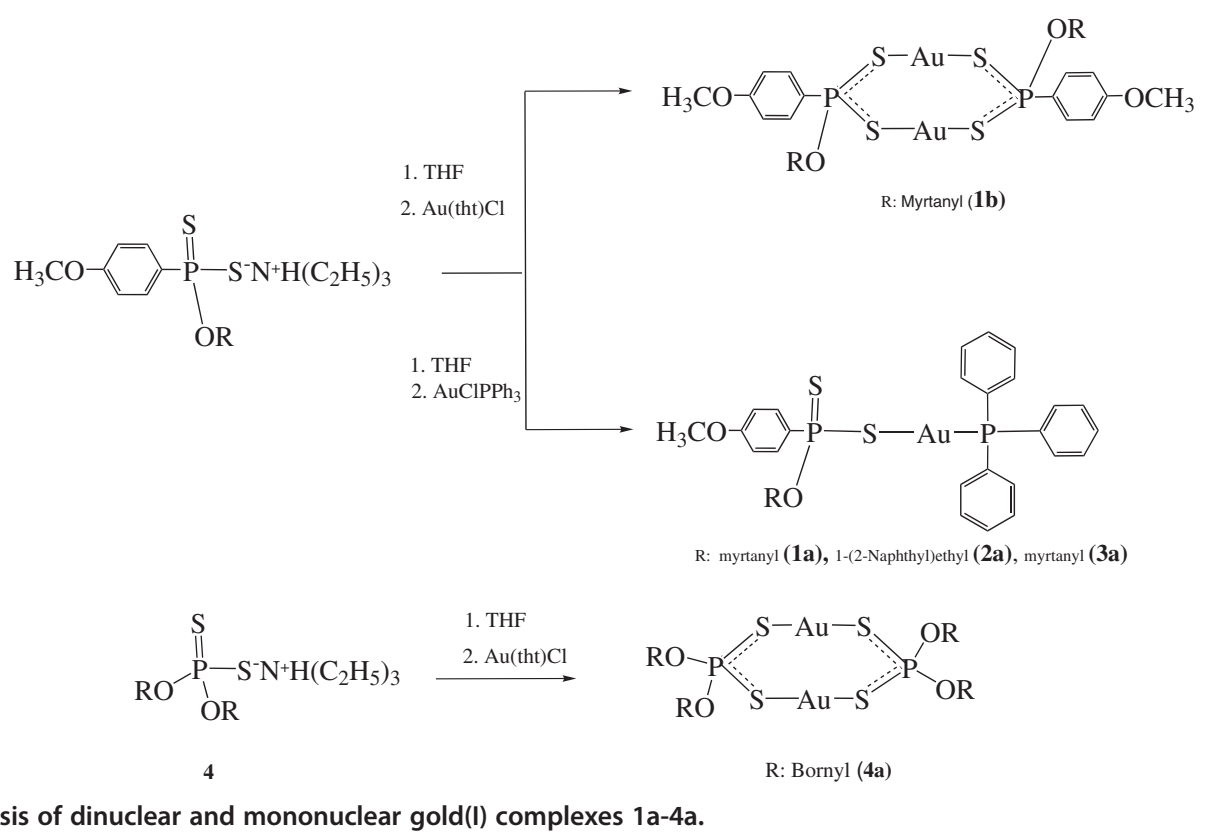

to obtain gold(I)-phosphin complexes with phosphorus1,1,-dithiolate (Scheme 2). The ${ }^{31} \mathrm{P}$ NMR spectrum of $\mathbf{1 a}$ exhibits two signal at $100.33 \mathrm{ppm}\left(\mathrm{PS}_{2}\right)$ and $37.30 \mathrm{ppm}$ $\left(\mathrm{PPh}_{3}\right)$. The complexes 2a and $3 \mathbf{a}$ also showed two signals in the ${ }^{31} \mathrm{P}$ NMR spectrum as expected and other spectroscopic data confirm their structure (Additional file 1). The reaction of the ligands $\mathbf{1}$ and $\mathbf{4}$ with $\mathrm{Au}$ (tht) $\mathrm{Cl}$ gave rise to dinuclear gold(I) complex $\mathbf{1 b}$ and $\mathbf{4 a}$. The spectra of the complexes $\mathbf{1 b}$ and $\mathbf{4 a}$ were suitable and agree with reported similar structures by Van Zyl et. al. [8-10,22]. To obtain dinuclear gold(I) complexes with amido derivatives of phosphoro-1,1-dithilato, all attempts were unsuccessful. The complex $\mathbf{1 b}$ displayed two signals as expexted in its ${ }^{31} \mathrm{P}$ NMR spectrum. One signal is assigned to the trans isomer and the other is assigned to cis isomer. Both signals in the ${ }^{31} \mathrm{P}$ NMR spectrum for dinuclear and mononuclear gold(I) complexes are slightly upfield when compared to the free ligands.

In the case of dinuclear silver(I) complexes, the ligands were reacted with $\mathrm{AgNO}_{3}$ in acetone-water(1:1) at room temperature (Scheme 3). The ${ }^{31} \mathrm{P}$ NMR spectra of the dinuclear silver(I) complexes are similar to the dinuclear gold(I) complexes. The expected cis and trans isomer were observed at 107.35 and $105.49 \mathrm{ppm}$ ( for 1c) and 107.2 and $105.8 \mathrm{ppm}$ (for $2 \mathbf{b}$ ) (Additional file 1).

The IR spectrum of the ligands and their complexes showed two characteristic bands at around 692-642 $\mathrm{cm}^{-1}$ and $582-515 \mathrm{~cm}^{-1}$ which are assigned to $v_{\mathrm{as}}\left(\mathrm{PS}_{2}\right)$ and $v_{\mathrm{s}}$ $\left(\mathrm{PS}_{2}\right)$, respectively (Additional file 1 ). The molecular ion peak of the ligands $\mathbf{2}$ and 4, the complexes $\mathbf{1 b}$ and $2 \mathbf{a}$ were observed at $\mathrm{m} / \mathrm{z}=430\left(\right.$ for $\left.[\mathrm{M}+1]^{+}\right), 476[\text { for } \mathrm{M}]^{+}, 832$ $\left(\right.$ for $\left.[\mathrm{M}]^{+}\right), 1104\left(\right.$ for $\left.[\mathrm{M}]^{+}\right)$, respectively, in their mass spectra whereas the other ligands and the complexes exhibited $\mathrm{m} / \mathrm{z}$-values for identifiable certain fragments (Additional file 1). Specific rotations of all compounds showed that only one optical isomer was formed.

\section{Molecular structure of 4}

Single crystals of $\mathbf{4}$ suitable for X-ray diffraction studies were obtained from acetone/ $n$-hexane. Compound 4 crystallizes in the orthorhombic space group P2(1)2(1)2(1) with $Z=4$. The molecular structure of $\mathbf{4}$ is depicted in Figure 1. Selected bond lengths and angles are given Table 1 and Crystal and structure-refinement data in Table 2. The P1-S1 and P1-S2 bond lengths are 197.56(5) and $198.13(5) \mathrm{pm}$, respectively. Those values are very<smiles>[R]OP(=S)(S[NH+]C(C)C)c1ccc(OC)cc1</smiles><smiles>C=O</smiles><smiles>[R20]OP(S[As])(=[SH][PH]([R20])(S[As])S[As])c1ccccc1</smiles>

R: myrtanyl (1c) and 1-(2-Naphthyl)ethyl (2b)

Scheme 3 Synthesis of dinuclear silver(I) complexes (1c and 2c). 


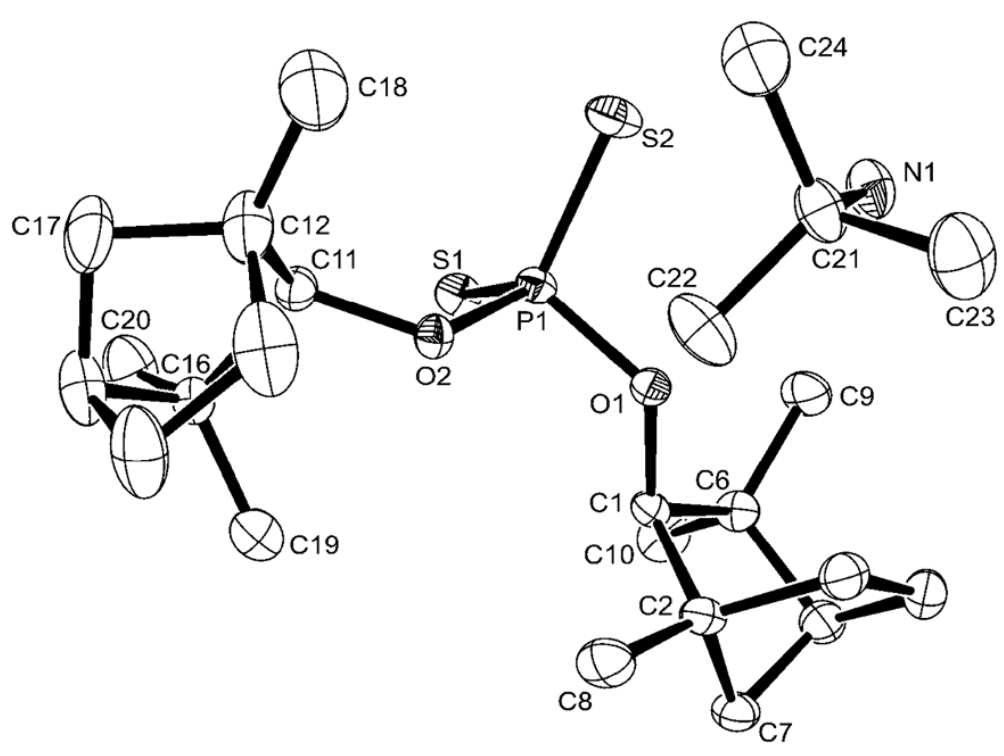

Figure 1 Molecular structure of 4 (ORTEP, 50\% probability).

close to each other due to a delocalized $\mathrm{PS}_{2}$ fragments. Bond lengths and angles are in a good agreement with those of related previously reported compounds [7,9]. Figure 1 shows that the phosphorus atom has a tetrahedral coordination environment with [O1-P1-S1 113.30(4) $)^{\circ}$, O2P1-S1 110.12(4) $)^{\circ}, \quad \mathrm{O}(1)-\mathrm{P}(1)-\mathrm{S}(2) \quad 105.02(4)^{\circ}, \quad \mathrm{O} 2-\mathrm{P} 1-\mathrm{S} 2$ $\left.110.05(4)^{\circ}\right]$ and the O1-P1-O2 $\left[99.61(6)^{\circ}\right]$ having a deviation from the ideal value $\left(109.5^{\circ}\right)$.

\section{Experimental}

\section{Materials}

When necessary, the reactions were carried out under an atmosphere of nitrogen using standard Schlenk techniques. All other chemicals were purchased from commercial sources and used directly without further purification.

\section{Measurements}

Elemental analyses were determined with a $\mathrm{GmbH}$ varioMICRO CHNS apparatus. Melting points were

\section{Table 1 Bond lengths [pm] and angles $\left[{ }^{\circ}\right]$ for 4}

\begin{tabular}{ll}
\hline$S(1)-P(1)$ & $197.56(5)$ \\
\hline$S(2)-P(1)$ & $198.13(5)$ \\
\hline$P(1)-O(1)$ & $159.14(11)$ \\
\hline$P(1)-O(2)$ & $160.96(11)$ \\
\hline$O(1)-P(1)-O(2)$ & $99.61(6)$ \\
\hline$O(1)-P(1)-S(1)$ & $113.30(4)$ \\
\hline$O(2)-P(1)-S(1)$ & $110.12(4)$ \\
\hline$O(1)-P(1)-S(2)$ & $105.02(4)$ \\
\hline$O(2)-P(1)-S(2)$ & $110.05(4)$ \\
\hline$S(1)-P(1)-S(2)$ & $117.23(2)$ \\
\hline
\end{tabular}

determined by using Electrotermal apparatus. NMR spectra were recorded on a Bruker AVANCE DRX 400 NMR spectrometer and Jeol GSX 270 in $\mathrm{CDCl}_{3}$ and $\mathrm{d}_{6}$ DMSO. IR spectra was measured on a Perkin-Elmer 2000 FTIR spectrophotometer $\left(4000-400 \mathrm{~cm}^{-1}\right)$. Mass spectra were recorded with an AGILENT 1100 MSD and Waters machines. Optical rotation values were determined with an automatic digital ADP 440+ polarimeter.

\section{X- ray crystallograpy}

Data were collected on an Xcalibur-S diffractometer (Agilent Technologies) using Mo- $K_{\alpha}$ radiation $(\lambda=$ $71.073 \mathrm{pm}$ ) and $\omega$-scan rotation (see Table 2). Data reduction was performed with CrysAlis Pro [32] including the program SCALE3 ABSPACK for empirical absorption correction. The structure was solved by direct methods and the refinement of all non-hydrogen atoms was performed with SHELX97 [33]. All non-hydrogen atoms were refined with anisotropic thermal parameters. With the exception of one borneol molecule $(\mathrm{C}(11)$ to $\mathrm{C}(20))$ all hydrogen atoms were located on difference Fourier maps calculated at the final stage of the structure refinement. The structure figure was generated with DIAMOND-3 [34]. Crystallographic details are given in the Additional file 2. CCDC (4) 921954 contains the supplementary crystallographic data for this paper. The data can be obtained free of charge from The Cambridge Crystallographic Data Centre via www.ccdc.cam.ac.uk/data_request/cif.

\section{Synthesis of compounds}

Synthesis of ${ }^{t-}$ butyl ammonium salt of $(1 S, 2 S, 5 S)-(-)$ myrtanyl -4- methoxyphenyl dithiophoshonate 1

2,4-Bis(4-methoxyphenyl)-1,3,2,4-dithiadiphosphetane-2,4disulfide (Lawesson's reagent: LR) ( $0.50 \mathrm{~g}, 1.23 \mathrm{mmol}$ ) was 
Table $\mathbf{2}$ Crystal data and structure refinement for $\mathbf{4}$

\begin{tabular}{|c|c|c|}
\hline Empirical formula & C24 H46 N O2 P S2 & \\
\hline Formula weight & 475.71 & \\
\hline Temperature & $130(2) \mathrm{K}$ & \\
\hline Wavelength & $71.073 \mathrm{pm}$ & \\
\hline Crystal system & Orthorhombic & \\
\hline Space group & $\mathrm{P} 2(1) 2(1) 2(1)$ & \\
\hline \multirow[t]{3}{*}{ Unit cell dimensions } & $\mathrm{a}=1029.440(10) \mathrm{pm}$ & $a=90^{\circ}$ \\
\hline & $\mathrm{b}=1366.64(2) \mathrm{pm}$ & $\beta=90^{\circ}$ \\
\hline & $\mathrm{c}=1949.83(2) \mathrm{pm}$ & $\delta=90^{\circ}$ \\
\hline Volume & $2.74316(6) \mathrm{nm} 3$ & \\
\hline Z & 4 & \\
\hline Density (calculated) & $1.152 \mathrm{Mg} / \mathrm{m} 3$ & \\
\hline Absorption coefficient & $0.272 \mathrm{~mm}-1$ & \\
\hline$F(000)$ & 1040 & \\
\hline Crystal size & $0.5 \times 0.4 \times 0.3 \mathrm{~mm} 3$ & \\
\hline $\begin{array}{l}\text { Theta range for } \\
\text { data collection }\end{array}$ & 2.98 to $30.51^{\circ}$ & \\
\hline Index ranges & $\begin{array}{l}-14<=\mathrm{h}<=14, \\
-19<=\mathrm{k}<=19 \\
-27<=\mid<=27\end{array}$ & \\
\hline Reflections collected & 56268 & \\
\hline Independent reflections & $8360[\mathrm{R}(\mathrm{int})=0.0375]$ & \\
\hline $\begin{array}{l}\text { Completeness } \\
\text { to theta }=30.51^{\circ}\end{array}$ & $99.8 \%$ & \\
\hline Absorption correction & $\begin{array}{l}\text { Semi-empirical from } \\
\text { equivalents }\end{array}$ & \\
\hline Max. and min. transmission & 1 and 0.98374 & \\
\hline Refinement method & $\begin{array}{l}\text { Full-matrix } \\
\text { least-squares on F2 }\end{array}$ & \\
\hline Data / restraints / parameters & 8360 / 0 / 390 & \\
\hline Goodness-of-fit on F2 & 0.997 & \\
\hline Final R indices [I > 2sigma(I)] & $R 1=0.0348, w R 2=0.0909$ & \\
\hline $\mathrm{R}$ indices (all data) & $\mathrm{R} 1=0.0403, w R 2=0.0925$ & \\
\hline Absolute structure parameter & $0.01(5)$ & \\
\hline Largest diff. peak and hole & 0.626 and -0.309 e. $. \AA-3$ & \\
\hline
\end{tabular}

reacted with 1S,2S,5S)-(-)-myrtanol (0.39g, $2.4693 \mathrm{mmol})$ in toluene $(20 \mathrm{~mL})$. The mixture was refluxed until all solids had dissolved. The colourless solution was cooled to rt, filtered and treated with excess tert-butyl amine. The product precipitated at $-18^{\circ} \mathrm{C}$ from toluene as a white solid, which was isolated by filtration, washed with toluene and $\mathrm{n}$-hexane and then dried in air. Yield: $0.76 \mathrm{~g}(72 \%), \mathrm{m}$. p.: $161-163^{\circ} \mathrm{C} .[\alpha]_{589}^{25}=57.14(\mathrm{c}=0.105$ in THF). Elemental analysis calculated for $\mathrm{C}_{21} \mathrm{H}_{36} \mathrm{NO}_{2} \mathrm{PS}_{2}\left(429.63 \mathrm{~g} \cdot \mathrm{mol}^{-1}\right): \mathrm{C}$, 58.71; H, 8.45; N, 3.26; S, 14.93, Found: C, 59.18; H, 8.51; $\mathrm{N}, 3.18 ; \mathrm{S}, 14.69 . \operatorname{IR}\left(\mathrm{cm}^{-1}\right): 670\left(v_{\text {asym }} \mathrm{PS}_{2}\right)$ and $556\left(v_{\text {sym }}\right.$ $\left.\mathrm{PS}_{2}\right) .{ }^{1} \mathrm{H}$ NMR $\left(\mathrm{CDCl}_{3}\right): \delta=8.07\left(\mathrm{dd}, 2 \mathrm{H},{ }^{3} \mathrm{~J}_{\mathrm{P}, \mathrm{H}}=13.65 \mathrm{~Hz}\right.$, $\left.{ }^{3} \mathrm{~J}_{\mathrm{H}, \mathrm{H}}=8.80 \mathrm{~Hz}\right), 6.9\left(\mathrm{dd}, 2 \mathrm{H},{ }^{4} \mathrm{~J}_{\mathrm{P} . \mathrm{H}}=2.57 \mathrm{~Hz},{ }^{3} \mathrm{~J}_{\mathrm{H} . \mathrm{H}}=\right.$ $8.75 \mathrm{~Hz}), 3.85(\mathrm{~s}, 3 \mathrm{H}, \mathrm{OCH} 3), 3.48\left(\mathrm{~m} .2 \mathrm{H}, \mathrm{OCH}_{2}\right), 1.88$ (m, 7H, myrtanyl), 1.37 (s, 9H.3×CH3), 1.17 (d, $3 \mathrm{H}, \mathrm{CH}_{3}$ ), 0.79(d, 3H, CH $\left.\mathrm{CH}_{3}\right)$ ppm. ${ }^{13} \mathrm{C}$ NMR $\left(\mathrm{CDCl}_{3}\right): \delta=161.32(\mathrm{~d}$, $\left.{ }^{4} \mathrm{~J}_{\mathrm{P}, \mathrm{C}}=2.91 \mathrm{~Hz}\right), 133.16\left(\mathrm{~d},{ }^{1} \mathrm{~J}_{\mathrm{P}, \mathrm{C}}=109.49 \mathrm{~Hz}\right), 132.32\left(\mathrm{~d},{ }^{2} \mathrm{~J}_{\mathrm{P}, \mathrm{C}}=\right.$ $13.43 \mathrm{~Hz}), 112.91\left(\mathrm{~d},{ }^{3} \mathrm{~J}_{\mathrm{P}, \mathrm{C}}=14.83 \mathrm{~Hz}\right), 68.78\left(\mathrm{~d},{ }^{2} \mathrm{~J}_{\mathrm{P}, \mathrm{C}}=8.55\right)$, 55.36, 54.00, 35.45(d, $\left.{ }^{3} \mathrm{~J}_{\mathrm{P}, \mathrm{C}}=8.40 \mathrm{~Hz}\right) 28.22,42.10,40.86$, 39.02, 26.63, 24.09, 23.36, 20.12, $18.12 \mathrm{ppm} .{ }^{31} \mathrm{P}$ NMR $\left(\mathrm{CDCl}_{3}\right): \delta=105.24 \mathrm{ppm}$. MS: $\mathrm{m} / \mathrm{z}=430[\mathrm{M}+1]^{+} .357[\mathrm{M}-$ $\left.\mathrm{NH}_{2} \mathrm{C}\left(\mathrm{CH}_{3}\right)_{3}\right]^{+}$.

\section{Triethyl ammonium salt of (S)-(-)-O-(2-naphthyl)ethyl-4-} methoxyphenyl dithiophosphonate 2

Compound 2 was prepared as described in the literature [7]. $[\alpha]_{589}^{25}=-37.74(\mathrm{c}=0.053$ in THF). Elemental analysis calculated for $\mathrm{C}_{25} \mathrm{H}_{34} \mathrm{NO}_{2} \mathrm{PS}_{2}$ (475.66 g.mol ${ }^{-1}$ ): C, 63.13; H, 7.21; N, 2.95; S, 13.48 \%, Found: C, 63.59; H, 7.32; N, 2.92; S, $12.74 \% . \mathrm{IR}\left(\mathrm{cm}^{-1}\right): 667\left(v_{\text {asym }} \mathrm{PS}_{2}\right)$ and $569\left(v_{\text {sym }}\right.$ $\left.\mathrm{PS}_{2}\right) .{ }^{1} \mathrm{H}-\mathrm{NMR}\left(\mathrm{CDCl}_{3}\right): \delta=9.89(\mathrm{br}, \mathrm{H}, \mathrm{HN}), 8.1(\mathrm{dd}, 2 \mathrm{H}$, $\left.{ }^{3} \mathrm{~J}_{\mathrm{P}, \mathrm{H}}=13.58 \mathrm{~Hz},{ }^{3} \mathrm{~J}_{\mathrm{H}, \mathrm{H}}=8.81 \mathrm{~Hz}\right), 7.21(\mathrm{~m} .4 \mathrm{H}$.), $7.53(\mathrm{dd}$, $1 \mathrm{H}), 7.40(\mathrm{~m} .2 \mathrm{H}), 6.7\left(\mathrm{dd}, 2 \mathrm{H} .{ }^{4} \mathrm{~J}_{\mathrm{P}, \mathrm{H}}=2.62 \mathrm{~Hz},{ }^{3} \mathrm{~J}_{\mathrm{H}, \mathrm{H}}=8.87\right.$ $\mathrm{Hz}), 5.7\left(\mathrm{~m} .1 \mathrm{H}, \mathrm{OCH},{ }^{3} \mathrm{~J}_{\mathrm{P}, \mathrm{H}}=13.07,{ }^{2} \mathrm{~J}_{\mathrm{H}, \mathrm{H}}=6.53 \mathrm{~Hz}\right), 3.69$ (s, 3H, OCH3), 3.07 (q, 6H, 3xNCH $\left.{ }_{2}\right), 1.56\left(\mathrm{~d}, 3 \mathrm{H}, \mathrm{CH}_{3}\right)$, $1.18\left(\mathrm{t}, 9 \mathrm{H}, 3 \times \mathrm{CH}_{3}\right) \mathrm{ppm} .{ }^{13} \mathrm{C} \mathrm{NMR}\left(\mathrm{CDCl}_{3}\right): \delta=160.75(\mathrm{~d}$, $\left.{ }^{4} \mathrm{~J}_{\mathrm{P}, \mathrm{C}}=3.03 \mathrm{~Hz}\right), 141.64\left(\mathrm{~d},{ }^{3} \mathrm{~J}_{\mathrm{P}, \mathrm{C}}=4.32 \mathrm{~Hz}\right), 135.54\left(\mathrm{~d},{ }^{1} \mathrm{~J}_{\mathrm{P}, \mathrm{C}}=\right.$ $111.36 \mathrm{~Hz}), 133.11,132.10,132.03\left(\mathrm{~d},{ }^{2} \mathrm{~J}_{\mathrm{P}, \mathrm{C}}=13.48 \mathrm{~Hz}\right)$, 127.97, 127.50, 125.70, 125.45, 124.99(d, $\left.{ }^{4} \mathrm{~J}_{\mathrm{P}, \mathrm{C}}=5.01 \mathrm{~Hz}\right)$, $112.52\left(\mathrm{~d},{ }^{3} \mathrm{~J}_{\mathrm{P}, \mathrm{C}}=14.88 \mathrm{~Hz}\right), 73.84\left(\mathrm{~d},{ }^{2} \mathrm{~J}_{\mathrm{P}, \mathrm{C}}=7.41\right), 55.23$, 45.90, 24.66 (d, $\left.{ }^{3} \mathrm{~J}_{\mathrm{P}, \mathrm{C}}=4.19 \mathrm{~Hz}\right), 8.48 \mathrm{ppm} .{ }^{31} \mathrm{P}$ NMR $\left(\mathrm{CDCl}_{3}\right): \delta=105.35 \mathrm{ppm}$.

\section{Synthesis of ${ }^{t-}$ Butyl ammonium salt of (1R)-(-)-O- myrtenyl-4- methoxyphenyl dithiophoshonate 3}

Compound 3 was prepared in the same way as compound 1, from LR (0.50 g, $1.23 \mathrm{mmol})$ and (1R)-(-)-myrtenol $(0.38 \mathrm{~mL} .2 .4693 \mathrm{mmol})$ in toluene $(20 \mathrm{~mL})$. Yield: $0.73 \mathrm{~g}$ (69\%), m.p.: $110-113^{\circ} \mathrm{C} .[\alpha]_{589}^{25}=159.1$ (c=0.044 in THF). Elemental analysis calculated for $\mathrm{C}_{21} \mathrm{H}_{34} \mathrm{NO}_{2} \mathrm{PS}_{2}$ (427.61 g.mol $\left.{ }^{-1}\right):$ C, 58.99; H, 8.01; N, 3.28; S, 15.00; Found: C, 59.18; H, 8.51; N, 3.18; S, 14.69. IR $\left(\mathrm{cm}^{-1}\right): 669\left(v_{\text {asym }} \mathrm{PS}_{2}\right)$ and $556\left(v_{\mathrm{sym}} \mathrm{PS}_{2}\right) .{ }^{1} \mathrm{H}$ NMR $\left(\mathrm{CDCl}_{3}\right): \delta=8.07\left(\mathrm{dd},{ }^{3} \mathrm{~J}_{\mathrm{P}, \mathrm{H}}=\right.$ $\left.13.59 \mathrm{~Hz},{ }^{3} \mathrm{~J}_{\mathrm{H}, \mathrm{H}}=8.83 \mathrm{~Hz}\right), 6.9\left(\mathrm{dd}, 2 \mathrm{H},{ }^{4} \mathrm{~J}_{\mathrm{P}, \mathrm{H}}=2.66 \mathrm{~Hz}\right.$, $\left.{ }^{3} \mathrm{~J}_{\mathrm{H}, \mathrm{H}}=8.88 \mathrm{~Hz}\right), 4.1\left(\mathrm{~m}, 2 \mathrm{H}, \mathrm{OCH}_{2}\right), 5.46(\mathrm{t}, \mathrm{H}, \mathrm{CH}), 3.84$ (s, $\left.3 \mathrm{H}, \mathrm{OCH}_{3}\right), 2.2\left(\mathrm{~m}, 5 \mathrm{H}\right.$, myrtenyl ), $1.38\left(\mathrm{~s}, 9 \mathrm{H}, 3 \times \mathrm{CH}_{3}\right)$, $1.27\left(\mathrm{~d}, 3 \mathrm{H}, \mathrm{CH}_{3}\right) 1.09(\mathrm{~d}, 1 \mathrm{H}, \mathrm{CH}) 0.74\left(\mathrm{~d}, 3 \mathrm{H}, \mathrm{CH}_{3}\right) \mathrm{ppm}$. ${ }^{13} \mathrm{C}$ NMR $\left(\mathrm{CDCl}_{3}\right): \delta=161.33\left(\mathrm{~d},{ }^{4} \mathrm{~J}_{\mathrm{P}, \mathrm{C}}=3.03 \mathrm{~Hz}\right), 144.20(\mathrm{~d}$, $\left.{ }^{3} \mathrm{~J}_{\mathrm{P}, \mathrm{C}}=9.68 \mathrm{~Hz}\right), 133.25\left(\mathrm{~d},{ }^{1} \mathrm{~J}_{\mathrm{P}, \mathrm{C}}=109.67 \mathrm{~Hz}\right), 132.29\left(\mathrm{~d},{ }^{2} \mathrm{~J}_{\mathrm{P}, \mathrm{C}}=\right.$ $13.50 \mathrm{~Hz}), 119.69,112.92\left(\mathrm{~d},{ }^{3} \mathrm{~J}_{\mathrm{P}, \mathrm{C}}=14.91 \mathrm{~Hz}\right), 67.70(\mathrm{~d}$, $\left.{ }^{2} \mathrm{~J}_{\mathrm{P}, \mathrm{C}}=7.62\right), 55.36,53.17,43.25,40.76,37.98,31.35,28.19$, 26.16, 20.97 ppm. ${ }^{31} \mathrm{P}$ NMR (CDCl3): $\delta=104.88 \mathrm{ppm}$.

\section{Synthesis of t'Butyl ammonium salt of $0,0^{\prime}$-dibornyl-4- methoxyphenyl dithiophosphonate (4)}

$\mathrm{P}_{4} \mathrm{~S}_{10}$ (1g, $2.25 \mathrm{mmol}$ ) was reacted with (-) borneol $(2.77 \mathrm{~g}, 9 \mathrm{mmol})$ in a $1: 4$ ratio in $50 \mathrm{~mL}$ hot toluene to give the crude dithiophosphoric acid. The reactions were refluxed until all solids dissolved and the yellow 
solutions were obtained. The solution was filtered and then was treated with excess tert-butyl amine at r.t. The tert-butyl ammonium salt of $\mathrm{O}, \mathrm{O}^{\prime}$-dibornyl-dithiophosphoric acids resulted in as a precipitated white solid product. The product was filtrated, washed with pentane several times, dried under vacuum and recrystallized from acetone/hexane. Yield: $3.89 \mathrm{~g}(91 \%)$, m.p.: $188^{\circ} \mathrm{C}$. Elemental analysis calculated for $\mathrm{C}_{24} \mathrm{H}_{46} \mathrm{NOPS}_{2}(475.73 \mathrm{~g} / \mathrm{mol})$ : C, 60.79; H, 9.26; N, 2.89; S, 13.18; Found: C, 60.59; H, 9.74; $\mathrm{N}, 2.94 ; \mathrm{S} 13.48 . \mathrm{IR}\left(\mathrm{cm}^{-1}\right): 665\left(v_{\text {asym }} \mathrm{PS}_{2}\right)$ and $560\left(v_{\text {sym }}\right.$ $\left.\mathrm{PS}_{2}\right) .{ }^{1} \mathrm{H}-\mathrm{NMR}\left(\mathrm{CDCl}_{3}\right): \delta=4.64(\mathrm{t}, 2 \mathrm{H}), 2.28(\mathrm{br}, 4 \mathrm{H})$, 2.00 (br, 2H), 1.71-179(br, m, 4H), 1.23(br, m, 4H), 0.91(s, $\left.6 \mathrm{H}, 2 \times \mathrm{CH}_{3}\right), 0.86\left(\mathrm{~s}, 6 \mathrm{H}, 2 \times \mathrm{CH}_{3}\right), 0.85\left(\mathrm{~s}, 6 \mathrm{H}, 2 \times \mathrm{CH}_{3}\right) .{ }^{13} \mathrm{C}-$ $\operatorname{NMR}\left(\mathrm{CDCl}_{3}\right): \delta=53.83,49.52\left(\mathrm{~d},{ }^{2} \mathrm{~J}_{\mathrm{P}, \mathrm{C}}=6.74 \mathrm{~Hz}\right), 47.31$, 44.93, 37.44, 28.30, 27.00, 19.94, 18.69, 13.67. ${ }^{31} \mathrm{P}-\mathrm{NMR}$ $\left(\mathrm{CDCl}_{3}\right): \delta=106.53(\mathrm{~d}) . \mathrm{MS}(\mathrm{FAB}): \mathrm{m} / \mathrm{z} 476[\mathrm{M}]^{+}$.

\section{Synthesis of complexes}

\section{$\left[\mathrm{Au}\left(\mathrm{PPh}_{3}\right)\left(\mathrm{R}^{1} \mathrm{PS}_{2}\left(\mathrm{OR}^{2}\right)\right]\left(R^{1}:\right.\right.$ 4-methoxyphenyl and $R^{2}$ :} $((1 S, 2 S, 5 S)-(-)-$ myrtanyl) $1 a$

A solution of the compound $1(0.044$ g. $0.102 \mathrm{mmol})$ in THF $(10 \mathrm{~mL})$ was added dropwise to a solution of $\mathrm{AuClPPh}_{3}(0.05 \mathrm{~g}, 0.102 \mathrm{mmol})$ in THF $(10 \mathrm{~mL})$ and stirred at r.t. for $2 \mathrm{~h}$. A colourless solution was observed and then a solid, t-butylammonium chloride, was immediately precipitated. The reaction mixture was filtered and the solvent was removed under reduced pressure. The reaction mixture was filtered and $10 \mathrm{~mL}$ of $\mathrm{n}$-hexane was added to the solution. The solvent was removed at room temperature and a white crystalline product was isolated. The white crystalline product was dried in air. Yield: $0.042 \mathrm{~g}(51 \%)$, m.p.: $72-74^{\circ} \mathrm{C} .[\alpha]_{589}^{25}=37.97(\mathrm{c}=0.079 \mathrm{in}$ THF). Elemental analysis calculated for $\mathrm{C}_{35} \mathrm{H}_{39} \mathrm{O}_{2} \mathrm{SP}_{2} \mathrm{Au}$ (814.78 g.mol ${ }^{-1}$ ); calcd: C, 51.60; H, 4.82; S, 7.87; Found: C, 49.78; H, 4.52; S, 10.61. IR $\left(\mathrm{cm}^{-1}\right): 668\left(v_{\text {asym }} \mathrm{PS}_{2}\right)$ and $536\left(v_{\text {sym }} \mathrm{PS}_{2}\right) .{ }^{1} \mathrm{H}$ NMR $\left(\mathrm{CDCl}_{3}\right): \delta=7.89\left(\mathrm{dd}, 2 \mathrm{H},{ }^{3} \mathrm{~J}_{\mathrm{P}, \mathrm{H}}=\right.$ $\left.13.77 \mathrm{~Hz},{ }^{3} \mathrm{~J}_{\mathrm{H}, \mathrm{H}}=8.61 \mathrm{~Hz}\right), 7.50(\mathrm{~m}, 15 \mathrm{H}),, 6.77(\mathrm{dd}, 2 \mathrm{H}$, $\left.{ }^{3} \mathrm{~J}_{\mathrm{H} . \mathrm{H}}=8.31 \mathrm{~Hz},{ }^{3} \mathrm{~J}_{\mathrm{P}, \mathrm{H}}=2.18 \mathrm{~Hz}\right), 3.95\left(\mathrm{q}, \mathrm{H}, \mathrm{OCH}_{2}\right), 3.73$ $\left(\mathrm{s}, 3 \mathrm{H}, \mathrm{OCH}_{3}\right) 1.80-0.78$ (br, $15 \mathrm{H}$, myrtanyl) ppm. ${ }^{31} \mathrm{P}$ $\operatorname{NMR}\left(\mathrm{CDCl}_{3}\right): \delta=100.33\left(\mathrm{PS}_{2}\right), 37.31\left(\mathrm{PPh}_{3}\right) \mathrm{ppm}$.

\section{$\left[A u\left\{R^{1} P S_{2}\left(O R^{2}\right)\right\}\right]_{2}\left(R^{1}:\right.$ 4-methoxyphenyl and $R^{2}$ : (1S,2S,5S)-(-)-myrtanyl) 16}

A solution of $\mathbf{1}(0.15 \mathrm{~g}, 0.35 \mathrm{mmol})$ in THF $(10 \mathrm{~mL})$ was added dropwise to a solution of $\mathrm{Au}($ tht) $\mathrm{Cl}$ (tht = tetrahydrothiophene) ( $0.11 \mathrm{~g}, 0.35 \mathrm{mmol})$ in THF $(10 \mathrm{~mL})$ and stirred for $2 \mathrm{~h}$. A solid, t-butylammonium chloride, was immediately observed. The reaction mixture was filtered and $10 \mathrm{~mL}$ of $\mathrm{n}$-hexane was added to the mixture. The solvent was removed at room temperature and orange crystals were isolated. Yield: $0.097 \mathrm{~g}(50 \%)$, m.p.: $123-125^{\circ} \mathrm{C} . \quad[\alpha]_{589}^{25}=29.85(\mathrm{c}=0.067$ in THF). Elemental analysis calculated for $\mathrm{C}_{34} \mathrm{H}_{48} \mathrm{O}_{4} \mathrm{~S}_{4} \mathrm{P}_{2} \mathrm{Au}_{2}\left(1104.90 \mathrm{~g} \cdot \mathrm{mol}^{-1}\right)$ : C, 36.96; H, 4.38; S, 11.61; Found: C, 36.76; H, 4.51; S,
11.75. IR $\left(\mathrm{cm}^{-1}\right): \quad 640\left(v_{\text {asym }} \mathrm{PS}_{2}\right)$ and $542\left(v_{\text {sym }} \quad \mathrm{PS}_{2}\right) .{ }^{1} \mathrm{H}$ $\operatorname{NMR}\left(\mathrm{CDCl}_{3}\right): \delta=7.91(\mathrm{br}, 4 \mathrm{H}), 6.90\left(\mathrm{dd}, 4 \mathrm{H},{ }^{3} \mathrm{~J}_{\mathrm{H}, \mathrm{H}}=8.63\right.$ $\left.\mathrm{Hz},{ }^{3} \mathrm{~J}_{\mathrm{P}, \mathrm{H}}=2.41 \mathrm{~Hz}\right), 4.40\left(\mathrm{br}, 4 \mathrm{H}, \mathrm{OCH}_{2}\right), 3.79(\mathrm{~s}, 6 \mathrm{H}$, $\left.\mathrm{OCH}_{3}\right), 1.81-1.29$ (m, $18 \mathrm{H}$. myrtenyl), $1.17\left(\mathrm{~s}, 6 \mathrm{H}, 2 \times \mathrm{CH}_{3}\right)$, $0.79\left(\mathrm{~s}, 6 \mathrm{H}, 2 \mathrm{xCH}_{3}\right) \mathrm{ppm} .{ }^{13} \mathrm{C}$ NMR $\left(\mathrm{CDCl}_{3}\right): \delta=163.06$ $\left(\mathrm{d},{ }^{4} \mathrm{~J}_{\mathrm{P}, \mathrm{C}}=3.12 \mathrm{~Hz}\right), 133.87\left(\mathrm{~d},{ }^{1} \mathrm{~J}_{\mathrm{P}, \mathrm{C}}=118.49 \mathrm{~Hz}\right), 132.46$ $\left(\mathrm{d},{ }^{2} \mathrm{~J}_{\mathrm{P}, \mathrm{C}}=13.25 \mathrm{~Hz}\right), 113.95\left(\mathrm{~d},{ }^{3} \mathrm{~J}_{\mathrm{P}, \mathrm{C}}=15.43 \mathrm{~Hz}\right), 71.31$ $\left(\mathrm{d},{ }^{2} \mathrm{~J}_{\mathrm{P}, \mathrm{C}}=5.33\right), 55.51,35.68\left(\mathrm{~d},{ }^{3} \mathrm{~J}_{\mathrm{P}, \mathrm{C}}=7.70 \mathrm{~Hz}\right), 42.23$, 40.84, 39.36, 28.20, 26.71, 24.03, 23.75, 20.28, 18.09 ppm. ${ }^{31} \mathrm{P}$ NMR $\left(\mathrm{CDCl}_{3}\right)$ : (cis, trans isomer) $\delta=104.12,100.95$. MS(ESI): $\mathrm{m} / \mathrm{z}=1104[\mathrm{M}]^{+}, 907[\mathrm{M}-\mathrm{Au}]^{+}$.

\section{$\left[A g\left\{R^{1} P S_{2}\left(O R^{2}\right)\right\}\right]_{2}\left(R^{1}:\right.$ 4-methoxyphenyl and $R^{2}$ : (1S,2S,5S)-(-)-myrtanyl) $1 c$}

The compound 1 ( $0.20 \mathrm{~g}, 0.41 \mathrm{mmol})$ was dissolved in acetone $(10 \mathrm{~mL})$. A solution of $\mathrm{AgNO}_{3}(0.08 \mathrm{~g}, 0.41 \mathrm{mmol})$ in a mixture $(10 \mathrm{~mL})$ of acetone-water $(1: 1)$ was added dropwise to the solution and stirred for $2 \mathrm{~h}$. A white powder solid was obtained, filtered and recrytallized in $\mathrm{CHCl}_{3}$. The solvent was removed at room temperature and white powder were isolated.Yield: $0.14 \mathrm{~g}$ (73\%), m.p.: $124-126^{\circ} \mathrm{C}$. $[\alpha]_{589}^{25}=27.39\left(\mathrm{c}=0.073\right.$ in $\left.\mathrm{CHCl}_{3}\right)$. Elemental analysis calculated for $\mathrm{C}_{34} \mathrm{H}_{48} \mathrm{O}_{4} \mathrm{~S}_{4} \mathrm{P}_{2} \mathrm{Ag}_{2}\left(926.70\right.$ g.mol $\left.{ }^{-1}\right)$ : C, 44.07; $\mathrm{H}, 5.22$; S, 13.84; Found: C, 43.62; H, 5.32; S, 13.37. IR $\left(\mathrm{cm}^{-1}\right)$ : $656\left(v_{\text {asym }} \mathrm{PS}_{2}\right)$ and $543\left(v_{\text {sym }} \mathrm{PS}_{2}\right) .{ }^{1} \mathrm{H}$ NMR $\left(\mathrm{CDCl}_{3}\right): \delta=$ $7.90\left(\mathrm{dd}, 4 \mathrm{H},{ }^{3} \mathrm{~J}_{\mathrm{P}, \mathrm{H}}=13.15 \mathrm{~Hz},{ }^{3} \mathrm{~J}_{\mathrm{H}, \mathrm{H}}=8.54 \mathrm{~Hz}\right), 6.84(\mathrm{br}$, $4 \mathrm{H}), 3.76\left(\mathrm{~s}, 6 \mathrm{H}, 2 \times \mathrm{OCH}_{3}\right), 1.9(\mathrm{~m}, 18 \mathrm{H}$, myrtanyl), 1.11 (d, $\left.6 \mathrm{H}, \mathrm{CH}_{3}\right) 0.73$ (d, $\left.6 \mathrm{H}, \mathrm{CH}_{3}\right)$ ppm. ${ }^{13} \mathrm{C}$ NMR $\left(\mathrm{CDCl}_{3}\right)$ : $\delta=162.24,132.50\left(\mathrm{~d},{ }^{2} \mathrm{~J}_{\mathrm{P}, \mathrm{C}}=13.24 \mathrm{~Hz}\right), 129.83\left(\mathrm{~d},{ }^{1} \mathrm{~J}_{\mathrm{P} . \mathrm{C}}=\right.$ $119.83 \mathrm{~Hz}), 113.54\left(\mathrm{~d},{ }^{3} \mathrm{~J}_{\mathrm{P}, \mathrm{C}}=15.06 \mathrm{~Hz}\right), 70.25\left(\mathrm{~d},{ }^{2} \mathrm{~J}_{\mathrm{P}, \mathrm{C}}=\right.$ 7.03), 55.42, 35.57 (d, ${ }^{3} \mathrm{~J}_{\mathrm{P}, \mathrm{C}}=8.40 \mathrm{~Hz}$ ), 42.19, 40.87, 39.22, 26.69, 24.11, 23.66, 20.54, 18.14 ppm. ${ }^{31} \mathrm{P}$ NMR $\left(\mathrm{CDCl}_{3}\right)$ (cis, trans isomer) $\delta=107.35$ and $105.49 \mathrm{ppm}$.

\section{$\left[\mathrm{Au}\left(\mathrm{PPh}_{3}\right)\left(\mathrm{R}^{1} P \mathrm{~S}_{2}\left(O \mathrm{R}^{2}\right)\right]\right.$ ( $\mathrm{R}^{1}$ : 4-methoxyphenyl, $R^{2}:((S)-(-)-O-$ (2-naphthyl)ethyl) $2 a$}

2a was prepared in a similar manner to 1a using $\mathrm{AuClPPh}_{3}(0.049$ g. $0.10 \mathrm{mmol})$ and t-butyl ammonium ((S)-(-)-O-(2-naphthyl)ethyl -4-methoxyphenydithiophosphonate $2(0.049$ g. $0.10 \mathrm{mmol})$. Yield: $0.04 \mathrm{~g}(48 \%)$, m.p.: $62-64{ }^{\circ} \mathrm{C} . \quad[\alpha]_{589}^{25}=51.94 \quad(\mathrm{c}=0.077$ in THF). Elemental analysis calculated for $\mathrm{C}_{37} \mathrm{H}_{33} \mathrm{O}_{2} \mathrm{~S}_{2} \mathrm{P}_{2} \mathrm{Au}\left(832.72\right.$ g.mol ${ }^{-1}$ ): C, 53.37; H, 3.99; S, 7.70; Found: C, 52.50; H, 4.12; S, $7.12 \%$. $\operatorname{IR}\left(\mathrm{cm}^{-1}\right): 674\left(v_{\text {asym }} \mathrm{PS}_{2}\right)$ and $537\left(v_{\text {sym }} \mathrm{PS}_{2}\right) .{ }^{1} \mathrm{H}$ NMR $\left(\mathrm{CDCl}_{3}\right): \delta=8.09\left(\mathrm{dd}, 2 \mathrm{H},{ }^{3} \mathrm{~J}_{\mathrm{P}, \mathrm{H}}=13.94 \mathrm{~Hz},{ }^{3} \mathrm{~J}_{\mathrm{H}, \mathrm{H}}=8.86\right.$ $\mathrm{Hz}$ ), 7.5(br, 15H), 6.79 (dd, $2 \mathrm{H},{ }^{3} \mathrm{~J}_{\mathrm{H} . \mathrm{H}}=8.86,{ }^{3} \mathrm{~J}_{\mathrm{P}, \mathrm{H}}=3.06$ $\mathrm{Hz}), 6.10(\mathrm{q}, \mathrm{H}, \mathrm{OCH}), 3.78\left(\mathrm{~s}, 3 \mathrm{H}, \mathrm{OCH}_{3}\right), 1.78(\mathrm{~m}, 3 \mathrm{H}$, $\left.\mathrm{CH}_{3}\right)$ ppm. ${ }^{13} \mathrm{C}$ NMR $\left(\mathrm{CDCl}_{3}\right): \delta=161.52\left(\mathrm{~d},{ }^{4} \mathrm{~J}_{\mathrm{P}, \mathrm{C}}=4.42\right.$ $\mathrm{Hz}), 135.21\left(\mathrm{~d},{ }^{1} \mathrm{~J}_{\mathrm{P} . \mathrm{C}}=134.60 \mathrm{~Hz}\right), 134.23\left(\mathrm{~d},{ }^{2} \mathrm{~J}_{\mathrm{P}, \mathrm{C}}=13.94\right.$ Hz), 132.07, 131.93, 131.70, 129.15, 128.14, 127.88, 127.56, $127.45,125.84,125.66,125.01,124.77,124.66,113.14$ (d, $\left.{ }^{3} \mathrm{~J}_{\mathrm{P}, \mathrm{C}}=15.63 \mathrm{~Hz}\right), 74.66\left(\mathrm{~d},{ }^{2} \mathrm{~J}_{\mathrm{P} . \mathrm{C}}=6.61 \mathrm{~Hz}\right), 55.30,24.82(\mathrm{~d})$ ppm. ${ }^{31} \mathrm{P} \mathrm{NMR}\left(\mathrm{CDCl}_{3}\right): \delta=100.43\left(\mathrm{PS}_{2}\right), 37.20\left(\mathrm{PPh}_{3}\right)$. $\mathrm{MS}(\mathrm{ESI})=\mathrm{m} / \mathrm{z}: 832[\mathrm{M}]^{+}$. 


\section{$\left[A g\left\{R^{1} P S_{2}\left(O R^{2}\right)\right\}\right]_{2}\left(R^{1}:\right.$ 4-methoxyphenyl and $R^{2}$ : (2-naphthyl) ethyl) $2 b$}

2b was prepared in a similar manner to $\mathbf{1 b}$ using $\mathrm{AgNO}_{3}$ $(0.07 \mathrm{~g}, 0.41 \mathrm{mmol})$ and triethyl ammonium(S)-(-)-O-(2naphthyl)ethyl-4-methoxyphenyl dithiophosphonate, $(0.20 \mathrm{~g}$, $0.41 \mathrm{mmol}$ ). Yield: $0.13 \mathrm{~g}(64 \%)$, m.p.: $114-115^{\circ} \mathrm{C} .[\alpha]_{589}^{25}=$ -36.14 ( $\mathrm{c}=0.083$ in $\mathrm{CHCl}_{3}$ ). Elemental analysis calculated for $\mathrm{C}_{38} \mathrm{H}_{36} \mathrm{O}_{4} \mathrm{P}_{2} \mathrm{~S}_{4} \mathrm{Ag}_{2}\left(962.65\right.$ g.mol $\left.{ }^{-1}\right): \mathrm{C}, 47.41 ; \mathrm{H}, 3.77 ; \mathrm{S}$, 13.32; Found: C, 46.15; H, 3.99; S, 12.29. IR $\left(\mathrm{cm}^{-1}\right): 655$ $\left(v_{\text {asym }} \mathrm{PS}_{2}\right)$ and $569\left(v_{\text {sym }} \mathrm{PS}_{2}\right) .{ }^{1} \mathrm{H}$ NMR $\left(\mathrm{CDCl}_{3}\right): \delta=7.60$ (br, 18H), 6.55 (br, 4H), 5.62 (br, $2 \mathrm{H}, \mathrm{OCH}), 3.55(\mathrm{~s}, 6 \mathrm{H}$, $\left.\mathrm{OCH}_{3}\right), 1.50\left(\mathrm{~d}, 6 \mathrm{H}, \mathrm{CH}_{3}\right) \mathrm{ppm} .{ }^{13} \mathrm{C} \mathrm{NMR}\left(\mathrm{CDCl}_{3}\right): \delta=$ $162.06\left(\mathrm{~d},{ }^{4} \mathrm{~J}_{\mathrm{P}, \mathrm{C}}=2.26 \mathrm{~Hz}\right), 139.78\left(\mathrm{~d},{ }^{4} \mathrm{~J}_{\mathrm{P}, \mathrm{C}}=4.27 \mathrm{~Hz}\right)$, $133.01\left(\mathrm{~d},{ }^{2} \mathrm{~J}_{\mathrm{P}, \mathrm{C}}=11.68 \mathrm{~Hz}\right), 129.94\left(\mathrm{~d},{ }^{1} \mathrm{~J}_{\mathrm{P}, \mathrm{C}}=117.92 \mathrm{~Hz}\right)$, $128.26,128.16,127.03,125.89,124.69 .124 .99\left(\mathrm{~d},{ }^{4} \mathrm{~J}_{\mathrm{P}, \mathrm{C}}=\right.$ $5.01 \mathrm{~Hz}), 113.22\left(\mathrm{~d},{ }^{3} \mathrm{~J}_{\mathrm{P}, \mathrm{C}}=12.57 \mathrm{~Hz}\right), 55.27,24.66$ (d) ppm. ${ }^{31} \mathrm{P}$ NMR $\left(\mathrm{CDCl}_{3}\right)$ (cis, trans isomer): $\delta=107.21$, 105.84 ppm. MS (ESI) $=$ m/z: 855[M-Ag] $]^{+}$.

\subsection{6. $\left[\mathrm{Au}\left(\mathrm{PPh}_{3}\right)\left(\mathrm{R}^{1} P S_{2}\left(\mathrm{OR}^{2}\right)\right]\left(R^{1}\right.\right.$ : 4-methoxyphenyl and $R^{2}$ : (1R-(-)-myrtenyl) $3 a$}

3a was prepared in a similar manner to 1a using $\mathrm{AuClPPh}_{3}(0.05$ g. $0.10 \mathrm{mmol}$ ) and t-butyl ammonium(1R)-(-)-myrtenyl-4-methoxyphenyldittiophosphonate $\mathbf{3}$ $(0.048$ g, $0.10 \mathrm{mmol})$. Yield: 0.036 g. $(43 \%)$, m.p.: $76-78^{\circ} \mathrm{C}$. $[\alpha]_{589}^{25}=30.76$ ( $c=0.065$ in THF). $\mathrm{C}_{35} \mathrm{H}_{37} \mathrm{O}_{2} \mathrm{SP}_{2} \mathrm{Au}(812.73$ g.mol ${ }^{-1}$ ); calcd: C, $51.72 ; \mathrm{H}, 4.59 ; \mathrm{S}, 7.89 \%$, found: C, 51.01; H, 5.22; S, 6.69\%. IR $\left(\mathrm{cm}^{-1}\right): 691\left(v_{\text {asym }} \mathrm{PS}_{2}\right)$ and 565 $\left(v_{\text {sym }} \mathrm{PS}_{2}\right) .{ }^{1} \mathrm{H}$ NMR $\left(\mathrm{CDCl}_{3}\right): \delta=7.99\left(\mathrm{dd}, 2 \mathrm{H},{ }^{3} \mathrm{~J}_{\mathrm{P} . \mathrm{H}}=\right.$ $\left.13.84 \mathrm{~Hz},{ }^{3} \mathrm{~J}_{\mathrm{H} . \mathrm{H}}=8.76 \mathrm{~Hz}\right), 7.41-7.19(\mathrm{br}, 15 \mathrm{H}$, ), $6.75(\mathrm{dd}$, $\left.2 \mathrm{H},{ }^{3} \mathrm{~J}_{\mathrm{H}, \mathrm{H}}=8.79 \mathrm{~Hz},{ }^{3} \mathrm{~J}_{\mathrm{P}, \mathrm{H}}=2.96 \mathrm{~Hz}\right), 5.49(\mathrm{t}, \mathrm{H}, \mathrm{CH}), 4.52$ (q, $\left.\mathrm{H}, \mathrm{OCH}_{2}\right), 3.71\left(\mathrm{~s}, 3 \mathrm{H}, \mathrm{OCH}_{3}\right), 2.25-078(\mathrm{br}, 12 \mathrm{H}$, myrtenyl) ppm. ${ }^{13} \mathrm{C}$ NMR $\left(\mathrm{CDCl}_{3}\right): \delta=161.56\left(\mathrm{~s},{ }^{4} \mathrm{~J}_{\mathrm{P}, \mathrm{C}}=\right.$ $3.32 \mathrm{~Hz}), 144.22\left(\mathrm{~d},{ }^{3} \mathrm{~J}_{\mathrm{P}, \mathrm{C}}=9.87 \mathrm{~Hz}\right), 134.37\left(\mathrm{~d},{ }^{1} \mathrm{~J}_{\mathrm{P} . \mathrm{C}}=\right.$ $121.39 \mathrm{~Hz}), 132.29\left(\mathrm{~d},{ }^{2} \mathrm{~J}_{\mathrm{P}, \mathrm{C}}=13.50 \mathrm{~Hz}\right), 131.75,129.52$, 128.94, 120.26, $113.25\left(\mathrm{~d},{ }^{3} \mathrm{~J}_{\mathrm{P} . \mathrm{C}}=15.47 \mathrm{~Hz}\right), 66.96\left(\mathrm{~d},{ }^{2} \mathrm{~J}_{\mathrm{P}, \mathrm{C}}=\right.$ $6.27 \mathrm{~Hz}), \quad 55.34,43.32,40.86,38.10,31.20,26.19$, $21.20 \mathrm{ppm} .{ }^{31} \mathrm{P}$ NMR $\left(\mathrm{CDCl}_{3}\right): \delta=100.60\left(\mathrm{PS}_{2}\right), 37.29$ $\left(\mathrm{PPh}_{3}\right) \mathrm{ppm}$.

\section{Synthesis of $\left[\mathrm{O}, \mathrm{O}^{\prime}-(\text { Bornyl) })_{2} \mathrm{PS}_{2}\right] \mathrm{Au}_{2} 4 a$}

Compound $4 \mathbf{a}$ was prepared in a similar manner to $\mathbf{1 b}$ using $4(0.075 \mathrm{~g}, 0.155 \mathrm{mmol})$ and $\mathrm{Au}($ tht $) \mathrm{Cl}$ (tht = tetrahydrothiophene) (0.05 g, $0.155 \mathrm{mmol})$ in $\operatorname{THF}(20 \mathrm{~mL})$. Yield: $0.065 \mathrm{~g}(\% 70)$, m.p. $212^{\circ} \mathrm{C}$. Elemental analysis calculated for $\mathrm{C}_{40} \mathrm{H}_{68} \mathrm{O}_{4} \mathrm{P}_{2} \mathrm{~S}_{4} \mathrm{Au}_{2}(1197.10 \mathrm{~g} / \mathrm{mol}): \mathrm{C}, 40.67 ; \mathrm{H}$, 5.68; S, 10.68; Found C, 40.13; H, 5.72; S, 10.71. IR $\left(\mathrm{cm}^{-1}\right)$ : 644 $\left(v_{\text {asym }} \mathrm{PS}_{2}\right)$ and $543\left(v_{\text {sym }} \mathrm{PS}_{2}\right) .{ }^{1} \mathrm{H}-\mathrm{NMR}\left(\mathrm{CDCl}_{3}\right): \delta=$ 4.95 (br, t, 2H), 2.43 (br, t, 2H), 1.79(br, s, 4H), 1.44(t, 4H, $\left.{ }^{3} \mathrm{~J}_{\mathrm{H}, \mathrm{H}}=\mathrm{Hz}\right), 1.30(\mathrm{br}, \mathrm{t}, 4 \mathrm{H}), 0.99\left(\mathrm{~s}, 6 \mathrm{H}, 2 \mathrm{xCH}_{3}\right), 0.93(\mathrm{~s}, 6 \mathrm{H}$, $\left.2 \times \mathrm{CH}_{3}\right), 0.89\left(\mathrm{~s}, 6 \mathrm{H}, 2 \mathrm{xCH}_{3}\right) .{ }^{13} \mathrm{C}-\mathrm{NMR}\left(\mathrm{CDCl}_{3}\right): \delta=49.94$, 47.55, 47.95, 37.35, 30.05, 27.96, 26.86, 19.91, 18.93, 13.71. ${ }^{31}$ P-NMR $\left(\mathrm{CDCl}_{3}\right): \delta=102.19(\mathrm{~s})$. MS (ESI): $\mathrm{m} / \mathrm{z} 999.5$ $[\mathrm{M}-\mathrm{Au}]^{+}, 967.5[\mathrm{M}-\mathrm{AuS}]^{+}$.

\section{Conclusion}

The new chiral phosphorus-1,1-dithiolate ligands were synthesized and were then utilised in the preparation of gold(I) and silver(I) phosphorus-1,1-dithiolate or S-donor with phosphine complexes. If the phosphorus-1,1dithiolate ligands carefully utilised in the preparation of gold(I) complexes, those type compounds can be improvement of the collecting metallic gold or silver from the minerals. We hope that this study will extend the unexplored area in phosphoro-1,1-dithiolate chemistry.

\section{Additional files}

Additional file 1: Spectra of the compounds.

Additional file 2: Cif file of compound 4.

\section{Competing interests}

The authors declare that they have no competing interests.

\section{Authors' contributions}

MK has coordinated the experimental work, characterized the structure of the all compounds and wrote the manuscript. SS synthesized the compounds and measured the IR spectra. CA synthesized and obtained the single crystals of the compound 4 . PL carried out X-ray studies. All authors have read and approved the final manuscript.

\section{Authors' information}

Part of M.Sc. of S. Solak.

\section{Acknowledgments}

This study was supported by Turkish Council of Research and Technology, TUBITAK (Grant no: 107T817) and Pamukkale University (Grant nos: 2009FBE020 and 2010FBE043).

\section{Author details}

'Department of Chemistry, Faculty of Arts \& Sciences, Pamukkale University, Kinikli 20075, Denizli, Turkey. ${ }^{2}$ Institut für Anorganische Chemie, Universität Leipzig, Johannisallee 29, D-04103, Leipzig, Germany.

Received: 12 February 2013 Accepted: 6 May 2013

Published: 20 May 2013

\section{References}

1. Burn AJ, Gosney I, Warrens CP, Wastle JP: Phosphorus-31 NMR investigation of the heterogeneous hydrolytic decomposition of zinc(II) bis(O, O-dialkyl dithiophosphate) lubricant additives. J Chem Soc Perkin Trans 1995, 2:265.

2. Contarini S, Tripaldi G, Ponti G, Lizzit S, Baraldi A, Paolucci G: Surface investigation of lubricant-metal interactions by synchrotron photoemission spectroscopy. App/ Surf Sci 1997, 108:359.

3. Fuller M, Yin Z, Kasrai M, Bancroft GM, Yamaguchi ES, Ryason PR, Willermet PA, Tan KH: Chemical characterization of tribochemical and thermal films generated from neutral and basic ZDDPs using X-ray absorption spectroscopy. Tribol Int 1997, 30:305.

4. Ma Q, Wang X-Y, Chen Q, Leung WH, Zhang QF: Dinuclear ruthenium complexes containing tripodal dithiophosphonate ligands. Inorg Chimica Acta 2011, 378:148.

5. Karakus M: Synthesis and Characterization of Chiral Gold (I) Phosphine Complexes with New Dithiophosphorus Ligands. Phosphorus Sulfur 2011 186:1523.

6. Karakus M, Lonnecke P, Hildebrand M, Hey-Hawkins E: Heterobimetallic Nickel(II) Complexes of Ferrocenyldithiophosphonates. Molecular Structures of [ $\{\mathrm{FCP}(\mathrm{OR}) \mathrm{S} 2\} 2 \mathrm{Ni}]\left[\mathrm{Fc}=\mathrm{Fe}(\eta 5-\mathrm{C} 5 \mathrm{H} 4)(\eta 5-\mathrm{C} 5 \mathrm{H} 5), \mathrm{R}=\mathrm{Et}, \mathrm{Pr}^{\mathrm{i}}, \mathrm{Bu}^{\mathrm{s}}, \mathrm{Bu}^{\mathrm{i}}\right]$. Z Anorg Allg Chem 2011, 637(7-8):983. 
7. Solak S, Karakus M, Tercan B, Hökelek T: Triethylammonium (S)-(-)-O-[1-(2naphthyl)ethyl] (4-methoxyphenyl)- dithiophosphonate. Acta Crystall Sec E-Structure Reports Online 2011, 67:01260.

8. Van Zyl WE: Dithiophosphonates and related P/S-type ligands of group 11 metals. Comments on Inorganic Chem 2010, 31:13.

9. Van Zyl WE, Lopez-de-Luzuriaga JM, Mohamed AA, Staples RJ, Fackler JP Jr: Dinuclear gold(I) dithiophosphonate complexes: Synthesis, luminescent properties, and X-ray crystal structures of $\left[\mathrm{AuS}_{2} \mathrm{PR}(\mathrm{OR})\right]_{2}\left(\mathrm{R}=\mathrm{Ph}, \mathrm{R}^{\prime}=\right.$ $\mathrm{C}_{5} \mathrm{H}_{5} ; \mathrm{R}=4-\mathrm{C}_{6} \mathrm{H}_{4} \mathrm{OMe}, \mathrm{R}^{\prime}=(1 \mathrm{~S}, 5 \mathrm{R}, 2 \mathrm{~S})-(-)$-menthyl; $\mathrm{R}=\mathrm{Fc}, \mathrm{R}^{\prime}=(\mathrm{CH} 2)_{2} \mathrm{O}$ $\left.\left(\mathrm{CH}_{2}\right)_{2} \mathrm{OMe}\right)$. Inorg Chem 2002, 11(17):4579.

10. Van Zyl WE, Staples RJ, Fackler JP Jr: Dinuclear gold(I) dithiophosphonate complexes: formation, structure and reactivity. Inorg Chem Commun 1998, I:51.

11. Pollnitz A, Silvestru A, Gimeno MC, Laguna A: New gold(I) and silver(I) complexes with organophosphorus ligands with SPNSO skeleton. Crystal and molecular structures of monomeric $\left[\mathrm{Au}\left\{\left(\mathrm{SPPh}_{2}\right)\left(\mathrm{O}_{2} \mathrm{SR}\right) \mathrm{N}\right\}\left(\mathrm{PPh}_{3}\right)\right](\mathrm{R}=$ $\left.\mathrm{Me}, \mathrm{C}_{6} \mathrm{H}_{4} \mathrm{Me}-4\right)$ and dimeric $\left.\left[\mathrm{Ag}\left\{\left(\mathrm{SPPh}_{2}\right)\left(\mathrm{O}_{2} \mathrm{SPh}\right) \mathrm{N}\right\}\left(\mathrm{PPh}_{3}\right)\right]_{2} \cdot 2 \mathrm{CH}_{2} \mathrm{Cl}_{2}\right]$. Inorg Chimica Acta 2010, 363:346.

12. Liu SL, Wang XY, Duan T, Leung WH, Zhang QF: Hydrolysis and coordination behavior of ferrocenyl-phosphonodithiolate: Synthesis and structure of $\mathrm{Cu}_{4}\left[\mathrm{FcP}\left(\mathrm{OCH}_{3}\right)(\mu-\mathrm{S})\left(\mu_{3}-\mathrm{S}\right)\right]_{4}\left[\mathrm{FC}=\mathrm{Fe}\left(\eta^{5}-\mathrm{C}_{5} \mathrm{H}_{4}\right)\left(\eta^{5}-\mathrm{C}_{5} \mathrm{H}_{5}\right)\right]$. J Mol Struct 2010, 964:78.

13. Alphia RG, Safin DA, Gimadiev TR, Pinus MV: Complexes of podandcontaining bis(dithiophosphonate)ligands with cobalt(II), nickel(II) and cadmium(II):recognation of $\mathrm{CH} 2 \mathrm{Cl} 2$. Transititon Met Chem 2008, 33:921.

14. Arsanious MHN, Maigali SS, Boulos LS: Scope and limitation $f$ the reaction of benzocycloalkanone oximes with Lawesson's reagents.

Phosphorus Sulfur 2010, 185:57.

15. Muniz J, Sansores LE, Martinez A, Salcedo R: Electronic structure and luminescence of $\left[\mathrm{AuS}_{2} \mathrm{PPh}\left(\mathrm{OCH}_{2} \mathrm{CH}=\mathrm{CH}_{2}\right)\right](2)$ complex. Theochem 2007, 820:141.

16. del Herna'ndez-Galindo MC, Jancik V, Moya-Cabrera MM, Toscano RA, CeaOlivares $\mathrm{R}$ : $2 \mathrm{D}$ hydrogen bond networks in the crystals of $\left[\left(\mathrm{NH}_{4}\right.\right.$ center dot $\mathrm{H} 2 \mathrm{O})(2)][(\mathrm{RO})(\mathrm{Fc}) \mathrm{P}(\mathrm{S})(2)](2)(\mathrm{R}=3-(\mathrm{BzO})-\mathrm{Bz}, 4-(\mathrm{n}-\mathrm{Bu})-\mathrm{Bz}, \mathrm{Bz}=$ benzyl). J Organomet Chem 2007, 692:5295.

17. Karakus M: Synthesis and Characterization of Novel Organothiophosphorus Compounds: X-ray Crystal Structure of $\mathrm{H}_{3} \mathrm{COC}_{6} \mathrm{H}_{4} \mathrm{P}\left(\mathrm{OC}_{2} \mathrm{H}_{4} \mathrm{~S}\right)(\mathrm{S})$ Synthesized by a New Method. $Z$ Anorg Allgs Chem 2006, 632(8-9):1549.

18. Foreman RSTJ, Slawin AMZ, Woollins JD: 2,4-Diferrocenyl-1,3, dithiadiphosphetane 2,4-disulfide; structure and reactions with catechols and $\left[\mathrm{PtCl}_{2}\left(\mathrm{PR}_{3}\right)_{2}\right](\mathrm{R}=\mathrm{Et}$ or $\mathrm{Bu})$. J Chem Soc Dalton Trans 1996:3653.

19. Foreman MRSJ, Slawin AMZ, Woollins JD: The reaction of dithiadiphosphetane disulfides with dienes, alkenesand thioaldehydes. J Chem Soc Dalton Trans 1999:1175.

20. Gray IP, Milton HL, Slawin AMZ, Woollins JD: Synthesis and Structure of [Fc(RO)PS $\left.{ }_{2}\right]^{-}$Complexes. Dalton Trans 2003:3450

21. Gray IP, Milton HL, Slawin AMZ, Woollins JD: Synthesis and Structure of [An(RO)PS $\left.]_{2}\right]^{-}$Complexes. Dalton Trans 2004:2477.

22. Maspero A, Kani I, Mohammed AA, Omary MA, Fackler RJ Jr: Syntheses and Structures of Dinuclear Gold(I) Dithiophosphonate Complexes and the Reaction of the Dithiophosphonate Complexes with Phosphines: Diverse Coordination Types. Inorg Chem 2003, 42:5311.

23. Karakus M, Aydogdu Y, Celik O, Kuzucu V, Ide S, Hey-Hawkins E: Synthesis, Molecular Structure, Optical Properties and Electrical Conductivity of Zwitterionic Ferrocenyldithiophosphonates. Z Anorg Allg Chem 2007, 633:405.

24. Haiduc I, Mezei G, Micu-Semeniuc R, Edelman FT, Fisher A: Differing Coordination Modes of (O-Alkyl)-p-Ethoxyphenyldithiophosphonato Ligands in Copper(I), Silver(I) and Gold(I) Triphenylphosphine Complexes. Z Anorg Allg Chem 2006, 632:295.

25. Albano VG, Aragoni MC, Arca M, Castellari C, Demartin F, Isaia F, Lippolis V, Loddo L, Verani G: An unprecendented example of a cis -phosphonodithioato nickel(II) complex built by an extensive hydrogen bonding supramolecular network. Commun: Chem; 2002:1170.

26. Santana MD, Garcia G, Navarro CM, Lozano AA, Perez J, Garcia L, Lopez G: Dithiophosphate and dithiophosphonate complexes of pentacoordinate nickel(II) containing the macrocycle 2,4,4-trimethyl-1,5,9triazacyclododec-1-ene ([12]aneN(3)-mc1) or its 9- methyl derivative $([12]$ aneN(3)-mc2). Crystal structures of [Ni([12]aneN(3)- mc1)\{S2P(OEt)
(2)\}][PF6] and [Ni([12]aneN(3)-mc1)\{S2P(p-CH3OPh)((OPr)-Pr-i)\}][PF6] Polyhedron 2002, 21:1935.

27. Karakus M, Alpoguz HK, Kaya A, Acar N, Gorgulu AO, Arslan M: A Kinetic Study of Mercury(II) Transport Through A Membrane Assisted by New Transport Reagent. Chem Cent J 2011, 5:45.

28. Aragoni MC, Arca M, Devillanova FA, Ferraro JR, Isaia F, Lelj F, Lippolis V, Verani G: An experimental and theoretical approach to phosphonodithioato complexes: molecular orbital analysis by hybridDFT and EHT calculations on trans-bis[O-alkylphenylphosphonodithioato] $\mathrm{Ni}^{\mathrm{II}}$, and vibrational assignments. Can J Chem 2001, 79:1483.

29. Karakus M, Lönnecke P, Hey-Hawkins E: Zwitterionic ferrocenyldithiophosphonates: the molecular structure of [FcP(S)S $\left.\left(\mathrm{OCH}_{2} \mathrm{CH}_{2} \mathrm{NH}_{2} \mathrm{Me}\right)\right]\left[\mathrm{Fc}=\mathrm{Fe}\left(\eta^{5}-\mathrm{C}_{5} \mathrm{H}_{4}\right)\left(\eta^{5}-\mathrm{C}_{5} \mathrm{H}_{5}\right)\right]$. Polyhedron 2004, 23:2281.

30. Baker MV, Barnard PJ, Berners-Price SJ, Brayshaw SK, Hickey JL, Skelton BW White $\mathrm{AH}$ : Synthesis and structural characterisation of linear $\mathrm{Au}(\mathrm{l})$ $\mathrm{N}$-heterocyclic carbene complexes: New analogues of the $\mathrm{Au}(\mathrm{I})$ phosphine drug Auranofin. J Organomet Chem 2005, 690:5625.

31. Ohta H, Kita M, Kanno H, Kojima M: Optically-active tris(O, O'-dialkyl dithiophosphato-S, S')Chromium(III) complexes. Isolation ofdiastereoisomers and isomerization reactions. Inorg Chim Acta 2000, 311:75.

32. CrysAlis Pro: Data collection and data reduction package, Agilent Technologies including the program SCALE3 ABSPACK for empirical absorption correction using spherical harmonics. Varian, Inc; 2006.

33. Sheldrick GM: SHELX includes SHELX-97, SHELXS-97. Acta Cryst 2008, A6 (4):112.

34. Brandenburg K: DIAMOND 3. Bonn, Germany: Crystal Impact Gbr.

\section{doi:10.1186/1752-153X-7-89}

Cite this article as: Solak et al.: Novel Gold(I) and Silver(I) Complexes of Phosphorus-1,1,-dithiolates and Molecular Structure of [O, $\mathrm{O}^{\prime}$-(Bornyl) $\left.{ }_{2} \mathrm{PS}_{2}\right] \mathrm{H}_{3} \mathrm{NC}\left(\mathrm{CH}_{3}\right)_{3}$. Chemistry Central Journal 2013 7:89.

Publish with ChemistryCentral and every
scientist can read your work free of charge
"Open access provides opportunities to our
colleagues in other parts of the globe, by allowing
anyone to view the content free of charge."
W. Jeffery Hurst, The Hershey Company.
- available free of charge to the entire scientific community
- peer reviewed and published immediately upon acceptance
- cited in PubMed and archived on PubMed Central
- yours - you keep the copyright
submit your manuscript here:
http://www.chemistrycentral.com/manuscript/

